\title{
THERMAL CREEP DEFORMATION IN PRESSURIZED THICK-WALLED FUNCTIONALLY GRADED ROTATING SPHERICAL SHELL
}

\author{
Sanjeev Sharma ${ }^{1 \S}$, Rekha Panchal ${ }^{2}$ \\ ${ }^{1,2}$ Department of Mathematics \\ Jaypee Institute of Information Technology \\ Noida, INDIA
}

\begin{abstract}
Creep stresses have been analyzed for pressurized thick-walled functionally graded rotating spherical shell with thermal effects. This paper aims to investigate the creep stresses in rotating functionally graded spherical shell. Transition theory using the concept of generalized measure has been used to investigate these creep stresses in rotating shell. From the numerical discussion, it has been observed that both rotation and non-homogeneity plays significant role on thermal creep stresses.
\end{abstract}

AMS Subject Classification: 74A10, 74C20, 74C99

Key Words: thermal, creep, spherical shell, FGM, pressure

\section{Introduction}

Functionally graded materials (FGMs) are non-homogeneous materials in composition, so that all its properties such as density, compressibility, Poisson's ratio and yield limit may vary throughout the material. There are many applications of functionally graded materials such as aerospace projects, medical industry, energy sector, nuclear projects and communication fields etc. Shells which keep rotating can be applied in engineering application such as high

Received: $\quad$ October 5, 2016

Revised: $\quad$ February 17, 2017

Published:

May 23, 2017 (c) 2017 Academic Publications, Ltd. url: www.acadpubl.eu

${ }^{\S}$ Correspondence author 
speed gears, composite rotors (flywheel) and turbine rotors. An advantage of spherical storage vessels is that their surface area per unit volume is smaller as compared to any other shape of vessel. Therefore, these types of vessels are important in solid mechanics and engineering applications. Non-homogeneity (functionally graded materials) can be arises due to certain external fields i.e. by thermal field, as the elastic module of the material changes with temperature or co-ordinates etc. Betton [1] discussed the creep behavior of thick-walled shells under pressure. Arya et al. [2] used large strain theory to investigate the impact of the material anisotropy on the creep of the thick-walled pressurized spherical vessels. Nezad et al. [3] determined stresses in thick functionally graded shell having exponentially variable properties subjected to pressure and concluded that non-homogeneity plays significant role on displacement and stress distribution. Sharma et al. [4] obtained the numerical solution for thermal elastic-plastic stresses in a functionally graded stainless steel composite cylinder with nonlinear strain hardening and Von-Mises' yield criterion using finite difference method and concluded that cylinder made of functionally graded stainless steel composite material with variable thickness and variable density under thermal loading is better choice of the design as compared to homogeneous cylinder. All these authors adopt the classical approach to investigate the stresses using the assumption of yield criterion, Norton's law. Transition theory with the concept of generalized strain measure [5] eliminates the use of above assumptions to investigate the stresses i.e. Gupta et al. [6] determined thermal creep stresses in thick isotropic spherical shell under internal pressure and concluded that shells made up of incompressible material requires high pressure to yield. Sharma et al. [7] investigated thermal creep stresses and concluded that thick-walled circular cylinder under external pressure is better for the designing purpose as compared to homogeneous cylinder. Sharma et al. [8] investigated thermal creep stresses for functionally graded thick-walled circular cylinder under pressure and concluded that functionally graded cylinder reduces the possibility of fracture of cylinder. With theoretical analysis and designing in the mind, this paper emphasize on the derivation of simple but reasonably accurate mathematical solutions to a wide range of shell problems. The objective of this paper is to study the effect of rotation on thermal creep stresses for thick-walled pressurized spherical shell made up of composite material using the concept of transition theory which eliminates the use of creep-strain law. Taking the non-homogeneity as the compressibility of the material in thick-walled spherical shells as,

$$
M=B_{0}\left(\frac{r}{b}\right)^{-t}
$$


where $a \leq r \leq b ; B_{0}$ and $t$ are constants.

Results obtained have been discussed numerically and represented graphically.

The generalized principal strain measure [5] is defined as

$$
\varepsilon_{i i}=\int_{0}^{\varepsilon_{i i}^{A}}\left[1-2 \varepsilon_{i i}^{A}\right]^{\frac{m}{2}-1} d \varepsilon_{i i}^{A}=\frac{1}{n}\left[1-\left(1-2 \varepsilon_{i i}^{A}\right)^{\frac{m}{2}}\right] .
$$

\section{Mathematical Formulation}

A thick-walled rotating spherical shell made up of composite material with inner radius $a$ and external radius $b$ is considered. The shell is subjected to internal and external pressure $p_{i}$ and $p_{e}$ respectively. The non-homogeneity in the shell is due to the variation of compressibility $M$. In spherical polar co-ordinates, the displacements are given as

$$
u=r(1-\kappa), v=0, w=0,
$$

where $\kappa$ is a function of $r$ only.

The generalized components of strain are,

$$
\varepsilon_{r r}=\frac{1}{n}\left[1-\left(r \kappa^{\prime}+\kappa\right)^{m}\right], \varepsilon_{\theta \theta}=\frac{1}{n}\left[1-\kappa^{m}\right]=\varepsilon_{\phi \phi}, \varepsilon_{r \theta}=\varepsilon_{\theta \phi}=\varepsilon_{\phi r}=0,
$$

where $n$ is the measure parameter and $\kappa^{\prime}=\frac{d \kappa}{d r}$.

The stress-strain relation with thermal effects for isotropic material is given as

$$
\tau_{i j}=\lambda \delta_{i j} I_{1}+2 \mu \varepsilon_{i j}-\xi T \delta_{i j},(i, j=1,2,3),
$$

where $I_{1}=\varepsilon_{k k}$ and $\tau_{i j}, \varepsilon_{i j}$ are stress and strain tensors respectively, $\delta_{i j}$ is Kronecker's delta, $\xi=\frac{3 \alpha}{M}, \lambda, \mu$ are Lame's constants and $T=\left(\frac{T 0 a}{b-a}\right)\left(\frac{b}{r}-1\right)$.

Equation of equilibrium is given by the expression

$$
\frac{d}{d r}\left(\tau_{r r}\right)+\frac{2\left(\tau_{r r}-\tau_{\theta \theta}\right)}{r}+\rho r \omega^{2}=0
$$

Using equation (4) and (5), equation (6) changes to non-linear differential equation in $\beta$ as

$$
Q(Q+1)^{m-1} \kappa \frac{d Q}{d \kappa}+Q(Q+1)^{m}-\frac{(4 M-3) M^{\prime} r}{(3-2 M) M \kappa^{m} m}\left[1-\kappa^{m}(Q+1)^{m}\right]
$$




$$
\begin{aligned}
+2(1-M) Q & -\frac{2 M}{m}\left[1-(Q+1)^{m}\right]-\frac{r\left[1-\kappa^{m}\right]}{\kappa^{m} m} \frac{\left[8 M M^{\prime}-4 M^{2} M-6 M^{\prime}\right]}{(3-2 M) M} \\
& +\alpha \frac{(3-2 M) r}{\kappa^{m} M}\left(M T^{\prime}-T M^{\prime}\right)-\frac{r\left(3 M-2 M^{2}\right) \rho r \omega^{2}}{3 \kappa^{m}}=0, \quad(7)
\end{aligned}
$$

where

$$
Q=\frac{r \kappa^{\prime}}{\kappa} \text { and } M=\frac{2 \mu}{(\lambda+2 \mu)} .
$$

The critical points of equation (7) are $Q \rightarrow-1$ and $Q \rightarrow \pm \infty$.

The boundary conditions considered for the problem are

$$
\tau_{r r}=-p_{i} \text { at } \mathrm{r}=\mathrm{a} ; \quad \tau_{\mathrm{rr}}=-\mathrm{p}_{\mathrm{e}} \text {, at } \mathrm{r}=\mathrm{b}
$$

\section{Method of Approach}

As material change its state from elastic to creep under some external loading and the asymptotic solution at each transition point gives the solution for the transition state. In this problem, we have considered only the principal stresses and it has been shown that [5-8] transition through $\tau_{r r}-\tau_{\theta \theta}$ results in creep state for the critical point $Q \rightarrow-1$. Thus transition function $R$ is defined as

$$
R_{1}=\tau_{r r}-\tau_{\theta \theta}=\frac{2 \mu \kappa^{m}}{m}\left[1-(Q+1)^{m}\right] .
$$

Taking asymptotic value of $\kappa$ as $Q \rightarrow-1$ and integrating after substituting equation (7) in logarithmic differentiation of equation (9), we have

$$
R_{1}=C_{1}(3-2 M) r^{-3 m} \exp x,
$$

where $C_{1}$ is constant of integration and

$$
x=2(1-m) \frac{B_{0}}{t}\left(\frac{r}{b}\right)^{-t}-X_{1}(r)-X_{2}(r)+X_{3}(r)-X_{4}(r),
$$

where

$$
\begin{gathered}
X_{1}(r)=\int \frac{1}{\kappa^{m}}\left(\frac{4 M M^{\prime}-3 M^{\prime}}{3 M-2 M^{2}}\right) d r \\
X_{2}(r)=\int\left(\frac{1}{\kappa^{m}}-1\right)\left(\frac{8 M M^{\prime}-4 M^{2} M^{\prime}-6 M^{\prime}}{3 M-2 M^{2}}\right) d r,
\end{gathered}
$$




$$
X_{3}(r)=\int \frac{m \alpha(3-2 M)\left(M T^{\prime}-T M^{\prime}\right)}{r \kappa^{m} M} d r
$$

and

$$
\mathrm{X}_{4}(r)=\int \frac{m}{\kappa^{m}} \frac{\left(3 M-2 M^{2}\right) \rho r \omega^{2}}{3} d r .
$$

With the use of asymptotic value of $\kappa$ as $Q \rightarrow-1\left(\kappa=\frac{D}{r}, D\right.$ being a constant), we get

$$
\begin{gathered}
X_{1}(r)=\int \frac{r^{m}\left(4 M M^{\prime}-3 M^{\prime}\right)}{D^{m}\left(3 M-2 M^{2}\right)} d r \\
X_{2}(r)=\int\left(\frac{r^{m}}{D^{m}}-1\right) \frac{\left(-4 M^{2} M^{\prime}+8 M^{\prime}-6 M^{\prime}\right)}{\left(3 M-2 M^{2}\right)} d r, \\
X_{3}(r)=\int \frac{\alpha m r^{m}\left(M T^{\prime}-T M^{\prime}\right)(3-2 M)}{D^{m} M} d r
\end{gathered}
$$

and

$$
X_{4}(r)=\int \frac{m r^{m}\left(3 M-2 M^{2}\right) \rho r \omega^{2}}{3 D^{m}} d r .
$$

From equations (9) and (11), it is found that

$$
\tau_{r r}-\tau_{\theta \theta}=C_{1} r X, X=(3-2 M) r^{-3 m-1} \exp x .
$$

Using the value of $\tau_{r r}-\tau_{\theta \theta}$ from equation (6) and equation (12), we get

$$
\tau_{r r}=C_{2}-2 C_{1} \int X d r-\frac{\rho r^{2} \omega^{2}}{2},
$$

where $C_{2}$ is a constant of integration.

The constants $C_{1}$ and $C_{2}$ are obtained by using the boundary conditions (8) as

$$
\begin{gathered}
C_{1}=\frac{p_{e}-p_{i}+\frac{\rho \omega^{2}\left(b^{2}-a^{2}\right)}{2}}{2 \int_{a}^{b} X d r}, \\
C_{2}=\frac{\left(p_{e}-p_{i}+\frac{\rho \omega^{2}\left(b^{2}-a^{2}\right)}{2}\right)}{\int_{a}^{b} X d r}\left[\int X d r\right]_{r=b}+\frac{\rho \omega^{2} b^{2}}{2}-p_{e} .
\end{gathered}
$$

As spherical shell is made up of composite material due to variable compressibility $M$ given by equation (1), the thermal creep stresses in a pressurized 
rotating shell becomes

$$
\begin{aligned}
& \tau_{r r}=-p_{e}+\frac{\rho \omega^{2}\left(b^{2}-r^{2}\right)}{2}+C_{3} \int_{r}^{b} X_{1} d r \\
& \tau_{\theta \theta}=-p_{e}+\frac{\rho \omega^{2}\left(b^{2}-r^{2}\right)}{2}+C_{3}\left[\int_{r}^{b} X_{1} d r-r X_{1}\right]=\tau_{\phi \phi},
\end{aligned}
$$

where

$$
\begin{gathered}
C_{3}=\frac{p_{e}-p_{i}+\frac{\rho \omega^{2}\left(b^{2}-a^{2}\right)}{2}}{\int_{a}^{b} X_{1} d r}, \quad X_{1}=r^{-3 m-1}\left(3-2 B_{0}\left(\frac{r}{b}\right)^{-t}\right) \exp x_{1}, \\
x_{1}=[x]_{M=B_{0}(r / b)^{-t}} .
\end{gathered}
$$

Equation (14) gives the thermal creep stresses for a thick-walled spherical shell with variable compressibility.

Now we use the following dimensionless components in equation (14)

$$
R_{0}=\frac{a}{b}, R=\frac{r}{b}, \sigma_{r r}=\frac{\tau_{r r}}{E}, \sigma_{\theta \theta}=\frac{\tau_{\theta \theta}}{E}, P_{i}=\frac{p_{i}}{E}, P_{e}=\frac{p_{e}}{E}, \omega_{1}^{2}=\frac{\rho \omega^{2} b^{2}}{E} .
$$

The equation (14) in non-dimensional form can be written as

$$
\begin{aligned}
\sigma_{r r} & =\frac{\omega_{1}^{2}\left(1-R^{2}\right)}{2}+C_{4} \int_{R}^{1} X_{2} d R-P_{e}, \\
\sigma_{\theta \theta} & =\frac{\omega_{1}^{2}\left(1-R^{2}\right)}{2}+C_{4}\left(\int_{R}^{1} X_{2} d r-b R X_{2}\right)-P_{e}=\sigma_{\phi \phi},
\end{aligned}
$$

where

$$
\mathrm{C}_{4}=\frac{P_{e}-P_{i}+\frac{\omega_{1}^{2}\left(1-R_{0}^{2}\right)}{2}}{\int_{R_{0}}^{1} X_{2} d R}, X_{2}=\left[X_{1}\right]_{M=B_{0}(r / b)^{-t}}, x_{2}=\left[x_{1}\right]_{M=B_{0}(r / b)^{-t}} .
$$

Equation (15) represents thermal creep stresses for thick-walled composite rotating pressurized spherical shell.

\section{Numerical Discussion}

For calculating the creep stresses, the definite integral in equation (??) has been evaluated by taking $\mathrm{D}=1$. To observe the various parameters, figures 1-4 have been made for stresses against radii ratio for measure $\mathrm{N}=3$, 


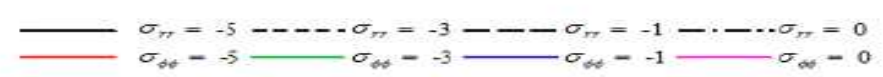

$\mathrm{K}($ Non-homogeneity parameter $)=-5,-3,-1,0 ; P_{1}=1.2, P_{2}=0.2$
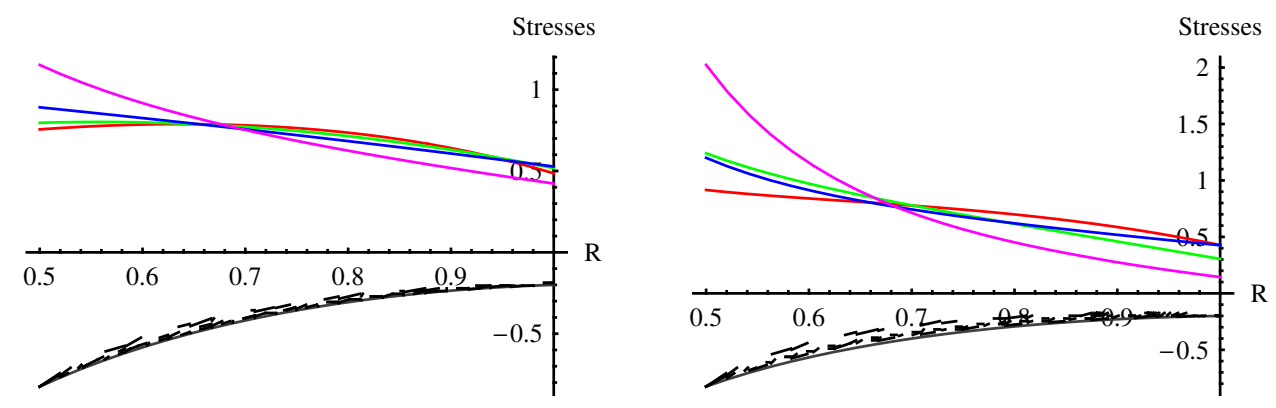

Figure 1: Thermal creep stresses in homogeneous/non-homogeneous rotating spherical shell under pressure with $(n=1 / 3), \theta_{1}=0,4$ and $\Omega^{2}=0.5$.

5 with angular speed $\omega_{1}^{2}=0.5,1$ at temperature $T_{1}=\alpha T_{0}=0,4$. Material used in this article is mild steel. Figures 1-2 have been drawn to discuss the influence of non-homogeneity and rotation on thermal creep stresses with different values of angular velocity for nonlinear measure when external pressure is less than that of internal pressure. Thermal circumferential stresses for non-homogeneous rotating spherical shell with an angular speed $\omega_{1}^{2}=0.5$ are maximum at internal surface with nonlinear measure $\mathrm{N}=3$ as can be seen from figure 1. Also, these stresses at room temperature are less at internal surface for less non-homogeneous spherical shell as compared to highly non-homogeneous spherical shell. Circumferential stresses for non-homogeneous spherical shell are tensile when thermal effects are introduced. Also, these thermal circumferential stresses are less for less non-homogeneous spherical shell as compared to highly non-homogeneous spherical shell. These circumferential stress increases significantly with the increase in temperature. With the increase in nonlinearity of the measure, circumferential stresses decreases significantly as can be seen from figure 2. Also, it has been noticed that circumferential stresses are less for non-homogeneous rotating spherical shell as compared to homogeneous rotating spherical shell. These circumferential stresses become tensile when temperature has been introduced. With increase in nonlinearity of the measure for $\mathrm{N}=3$ to $\mathrm{N}=5$ these circumferential stresses decreases significantly and are maximum at internal surface. When internal pressure is more than that 

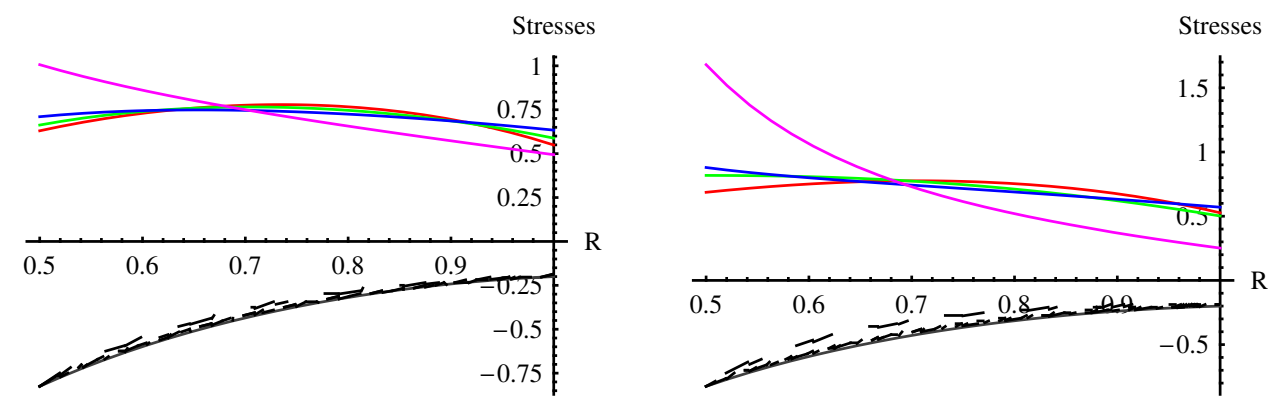

Figure 2: Thermal creep stresses in homogeneous/non-homogeneous rotating spherical shell under pressure with $(\mathrm{n}=1 / 5), \theta_{1}=0,4$ and $\Omega^{2}=0.5$.
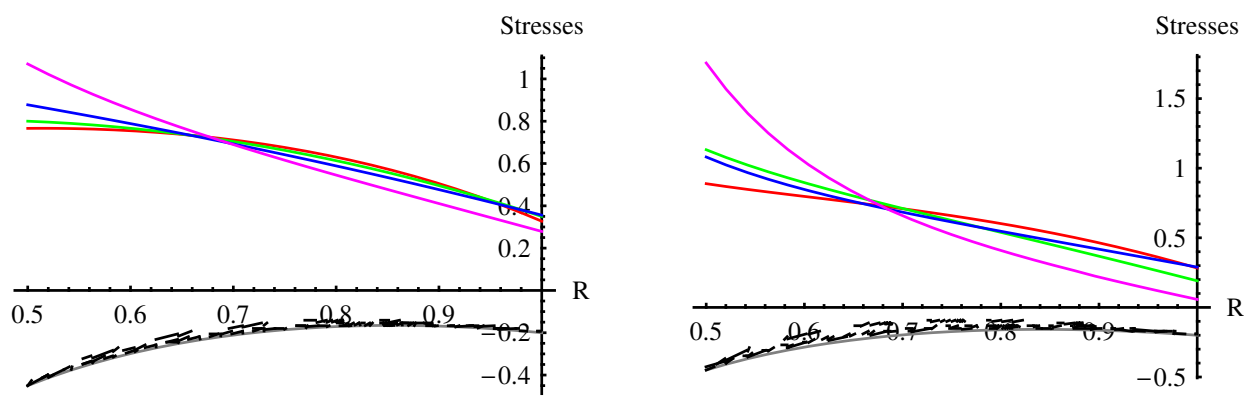

Figure 3: Thermal creep stresses in homogeneous/non-homogeneous rotating spherical shell under pressure with $(\mathrm{n}=1 / 3), \theta_{1}=0,4$ and $\Omega^{2}=1$.

of external pressure, thermal creep stresses in figures 3-4 with angular velocity $\Omega^{2}=1$ have been drawn for nonlinear measure. Circumferential stresses are tensile and maximum at internal surface for nonlinear measure $\mathrm{N}=3$ as can be seen from figure 3. Also, these thermal stresses are less at internal surface for less non-homogeneous rotating spherical shell as compared to highly non-homogeneous rotating spherical shell. These circumferential stresses increased with the introduction of temperature. Thermal circumferential stresses are minimum for less non-homogeneous rotating spherical shell as compared to highly non-homogeneous rotating spherical shell and these stresses decreased as the measure changes from 3 to 5 which can be seen from figure 4 . These thermal circumferential stresses are less at the external surface for homogeneous rotating shell as compared to non-homogeneous rotating shell. These thermal circumferential stresses decreased with the change in measure from $\mathrm{N}=3$ to $\mathrm{N}$ $=5$. 

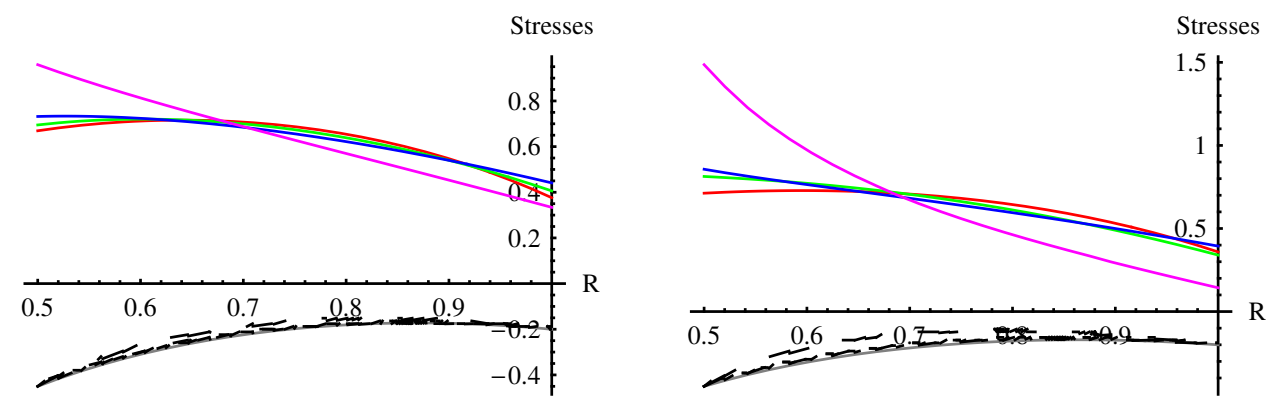

Figure 4: Thermal creep stresses in homogeneous/non-homogeneous rotating spherical shell under pressure with $(n=1 / 5), \theta_{1}=0,4$ and $\Omega^{2}=1$.

\section{Conclusion}

It is seen from the numerical discussion that both rotation and non-homogeneity wof the spherical shell have significant effect on creep stresses. Also, by introduction of a suitably chosen temperature gradient, nonlinear strain measure thick-walled spherical shell made of highly non-homogeneous material is on the safer side of the design for different values of $\mathrm{N}, \Omega^{2}$ and temperature as compared to less non-homogeneous material which leads to the idea of "Stress saving" and minimizing the likelihood of fracture of rotating spherical shell.

\section{References}

[1] J. Betton, Creep Mechanics, 3-rd edition, Springer, Germany, 2008.

[2] V. K. Arya, K. K. Debnath, N.S. Bhatnagar, The spherical vessel with creep properties considering large strains, Int. J. Press. Vess. Piping, 15 (1980), 185-193.

[3] Nezad Zamani, M. Zahra, H.T. Tahereh, N. Abbas, Closed-form analytical solution for creep stresses of pressurized functionally graded material thick spherical shells, Adv. Sci. Lett., 19, No. 2 (2013), 464-467.

[4] S. Sharma, S. Yadav, Thermo elastic-plastic analysis of rotating functionally graded stainless steel composite cylinder under internal and external pressure using finite difference method, Advances in Materials Science and Engineering, 2013, (2013), 1-10.

[5] B.N. Borah, Thermo elastic-plastic transition, Contemporary Mathematics, 379 (2005), 93-111.

[6] S.K. Gupta, Creep transition of a thick isotropic spherical shell under internal pressure, Indian J. Pure \& Appl. Math., 19 (1988), 1239-1248.

[7] S. Sharma, I. Sahai, R. Kumar, Creep Transition in Non Homogeneous Thick Walled Circular Cylinder under Internal and External Pressure, Applied Mathematical Science, 6 (2012), 6075080. 
[8] S. Sharma, A.K. Aggarwal, R. Sharma, Safety analysis of thermal creep non-homogeneous thick-walled circular cylinder under internal and external pressure using Lebesgue strain measure, Multidiscipline Modeling in Materials and Structures, 9 (2013), 499-513. 